\title{
Numerical Simulation of Blood Flow in Centrifugal Heart Pump by Utilizing Meshless Smoothed Particles Hydrodynamic Method
}

\author{
ErfanRahmanian $^{1}$ MehdiNavidbakhsh $^{2}$, Mohammad mohammadzadeh ${ }^{3}$, \\ Hamed Habibi ${ }^{4}$ \\ MSc, School of Biomechanical Engineering, Iran University of Science and \\ Technology, Tehran, Iran ${ }^{1,3}$ \\ Associate Professor, Iran University of Science and Technology, Tehran, Iran ${ }^{2}$ \\ MSc, School of Mechanical Engineering, College of Engineering University of \\ Tehran, Tehran, Iran ${ }^{4}$ \\ erfanrahmanian@mecheng.iust.ac.ir ${ }^{1}$,mnaviad@iust.ac.ir ${ }^{2}$ \\ m_mohammadzadeh@mecheng.iust.ac.ir ${ }^{3}$,hammed28160@gmail.com ${ }^{4}$
}

\begin{abstract}
Numerical methods have had a tremendous effect in optimization of heart devices. Among all methods meshing independentalgorithms, as modern methods in simulations, have solved most problems related to meshing and reduced its complexity. In this paper, blood flow in heart centrifugal pump is simulated by dynamic of particles and based on this model, governing equations of velocity field and stress distribution, are determined. Also, the harmful locations in which hemolysis and thrombosis occur, are detected. In the present work we explore the feasibility of performing computations of such flows using SPH.To evaluate the accuracy of the computations the results are compared to other researches in [7,4] .The analysis results can also be used as the basis for further researches and the improvement of centrifugal pump.
\end{abstract}

Keywords: Heart Pump, Hemolysis, Smooth particles hydrodynamic particles, Clotting, Numerical solution method

\section{Introduction}

Nowadays, ventricular assistance devices are used as a bridge to transplanting to help heart patients. It has been shown that more than 20 million people in the world suffer from heart diseases [1]. The number of patients who need heart transplant is extremely greater than donated organs..The progresses in heart pumps design resulted in more compatibility of these equipments with human physiological system. Numerical solution method and simulations have improved the heart pumps function and helped to eliminate the main problems relating to blood such as hemolysis and thrombosis. These problems are due to flows those are not consistent with natural blood circulation, as well as return flow and low speed regions of blood where the blood may be clotted. The red blood cells are potentially subject to huge shear stress in heart pumps, but due to limited applying time of this shear stress, the corresponding damage is considerably low. So, the pump designers are always trying to detect locations in which hemolysis and blood clotting are probable and increase compatibility to natural body physiologyby correcting the geometrical structure of pumps [6].The computational fluid dynamichas had deep influence on optimization of heart equipment devices for some decades. In general, there are three different attitudes to analyze hydraulic problems that are Lagrange, Euler and combination of these two methods. The essence of problems deeply effects on selection of analysis method. The present numerical methods that are mostly based 
on meshing, consumes a lot of time for these problems and numerical distributed errors that will be produced in Euler methods due to discretizing of transfer terms in Navier-Stokes equations, reduces solution accuracy. Therefore it can be concluded that Lagrange mesh less methods are appropriate alternatives to analyze hydraulic problems. In traditional methods, for instance finite element method or finite difference method, flow meshing is a very important step in analysis. In three dimensional and complex geometry problems, it is needed to spend a lot of time for domain discretizing by mesh. In addition, the meshing should be modified in each time step, if the problem consists of moving boundary. So, the non-meshing methods have been developed recently to overcome the above mentioned problems. This method was firstly used by Lucy in Astrophysics problems. The other pioneer researcher in this field is Monaghan, who utilized this method for analyzing the free surface flow and reached to acceptable results.

\section{Material and Methods}

The mass and momentum conservation equations that govern on fluid motion are as below:

$$
\begin{aligned}
& \frac{1}{\rho} \frac{D \rho}{D t}+\nabla \cdot \vec{u}=0 \\
& \frac{D \vec{u}}{D t}=-\frac{1}{\rho} \nabla P+g+\frac{1}{\rho} \nabla \cdot \vec{\tau}
\end{aligned}
$$

where $\rho$ is density, $u$ is velocity vector, $P$ is pressure and $\tau$ is stress tensor. Smooth particles hydro dynamic method is a weighted interpolate to estimate a quantity. Indeed to estimate a parameter at a given point, the weighted mean of corresponding values of neighbor points, will be calculated on a parabolic interpolation function basis. This neighborhood is said affected radius. With respect to basic concept of $\mathrm{SPH}$, the value of quantity $\mathrm{f}$ at this point is:

$$
f\left(r_{0}\right)=\int_{r 1}^{r 2} f(r) w\left(\left|r-r_{0}\right|, h\right) \cdot d r
$$

where $\mathrm{W}$ is interpolation function and $\mathrm{h}$ is smooth length that specifies the affected radius. In numerical methods, due to infinite points in affected radius, it is not practical to utilize Eq. 3 and actually some finite points will be $f_{a} \approx \sum_{b} m_{b} \frac{f_{b}}{\rho_{b}} w_{a b}$

where $\mathrm{W}_{\mathrm{ab}}=\mathrm{W}\left(\mathrm{r}_{\mathrm{a}}-\mathrm{r}_{\mathrm{b}}, \mathrm{h}\right)$ and $\mathrm{m}$ is number of neighbor particles and $\mathrm{b}$ is central particle. If $\mathrm{f}$ is replaced by $\mathrm{w}$ inequation (4) so it is changed to equation (5):

$$
\rho_{a} \approx \sum_{b} m_{b} w_{a b}
$$

\subsection{Interpolation Function}

The interpolation function has important role in SPH method because this function determines how to approximate a quantity and states the affected length for each particle. This interpolation function should satisfy the following conditions: If affected length is shown by $\Omega 1$ and out of affected length is shown by $\Omega 0$,so we have:
A: in $\Omega 1$
$\mathrm{w}(\mathrm{r}, \mathrm{h})>0$
B: in $\Omega 0$
$\mathrm{w}(\mathrm{r}, \mathrm{h})=0$ 
C: $\int w(r, h) \cdot d \Omega=1$

D.An interpolation function should be uniformly decreasing in $\Omega 1$ area as it maximizes at center and be zero at boundary. The two first conditions cause a point interacts with a limited number of its adjacent points and this enables us to make an approximation fora particle in a local definition. The third condition is needed for stability and the last condition states that close points to central point have greater impact on interpolation.

$$
\begin{aligned}
& w(r, h)=\frac{10}{7 \pi h^{2}}\left(1-\frac{3}{2} q^{2}+\frac{3}{4} q^{3}\right) \quad q<1 \\
& w(r, h)=\frac{10}{28 \pi h^{2}}(2-q)^{3} \quad 1<q<2 \\
& w(r, h)=0 \quad q>2
\end{aligned}
$$

where $\mathrm{q}=\mathrm{r} / \mathrm{h}$ and $\mathrm{r}$ is particle distance. Smooth length $\mathrm{h}$ determines the effective radius around particle a.

\subsection{Smooth Length}

The smooth length $\mathrm{h}$, in smooth particles hydrodynamic method, always states the effective area of this method. Maybe not enough particles exist in affected area, if $\mathrm{h}$ is too small. This condition extremely reduces the accuracy. If the $h$ is too long then all details of particles and local characteristicsmay be no smooth anymore. This will have negative effects on result and calculation volume will be increased dramatically. Therefore this parameter should be chosen carefully. Smooth length $h$ determines the effective radius around particle a and directly depends on fluid density.In incompressible fluids, such as blood, a constant smooth length can be selected for all points and times.

\subsection{Derivation in SPH Method}

In SPH method the derivation is generally applied on interpolation function. Therefore the derivation is defined as below

$$
\begin{aligned}
& f_{a} \approx \sum_{b} m_{b} \frac{f_{b}}{\rho_{b}} W_{a b} \\
& \nabla f_{a} \approx \sum_{b} m_{b} \frac{f_{b}}{\rho_{b}} \nabla \mathcal{W}_{a b}
\end{aligned}
$$

Where $\nabla$ is gradient with respect to central particle a. The derivation of pressure term in momentum equation by using chain rule, is as below

$$
\left(\frac{\nabla P}{\rho}\right)=\nabla\left(\frac{P}{\rho}\right)+\frac{P}{\rho^{2}} \nabla \rho
$$

Considering the results of chain derivation, the derivation of interpolation function between two particles $\mathrm{a}, \mathrm{b}$ will be:

$$
\left(\frac{\nabla P}{\rho}\right)=\sum_{b} m_{b} \frac{P_{b}}{\rho_{b}^{2}} \nabla_{a} W_{a b}+\frac{P}{\rho_{a}^{2}} \sum_{b} m_{b} \nabla_{a} w_{a b}
$$


Similarly for a vector quantity we write:

$$
\left(\frac{\nabla P}{\rho}\right)=\sum_{b} m_{b}\left(\frac{P_{b}}{\rho_{b}^{2}}+\frac{P_{a}}{\rho_{a}^{2}}\right) \nabla_{a} w_{a b}
$$

\subsection{General algorithm of solution}

Firstly, the smooth length $h$ and the particles coordinates should be determined according to the scale of problem and the number of particles. Considering the density, smooth length $h$ and mass of all particles is determined by equation (4). Also, at this step, the velocity of all particles is regulated considering the initial conditions.

In prediction stage, ignoring the pressure term in Eq. 2, the particles situation and velocity in next time step, is calculated as below:

$$
\begin{aligned}
& \Delta u_{*}=\left(g+\frac{\mu}{\rho} \nabla^{2} \bar{u}\right) \Delta t \\
& \bar{u}_{*}=u_{t}+\Delta u_{*} \\
& \vec{r}_{*}=\vec{r}_{t}+\vec{u}_{*} \Delta t
\end{aligned}
$$

where $u_{t}$ and $r_{t}$ are velocities and locations of particles at current time step and $\Delta \mathrm{u}^{*}$ is variation of velocity of fluid at prediction step, $\mathrm{r}^{*}$ and $\mathrm{u}^{*}$ are predicted values of velocity and location of particles in next time step respectively and $\Delta \mathrm{t}$ is time step.

$\rho^{*}$ is density and will be determined by predicted location of particles $\mathrm{r}^{*}$ and Eq. (5). $\rho^{*}$ is different from $\rho_{0}$ due to ignoring pressure term in momentum equation. The fluid pressure will be estimated in next stage and be used in momentum equation to satisfy incompressible fluid condition. The obtained equation, using the estimated pressure, is mass conservation equation.

$$
\frac{1}{\rho} \frac{\rho_{0}-\rho_{*}}{\Delta t}+\nabla\left(U_{* *}\right)=0
$$

where $\rho_{*}$ is particles density in prediction step, $\rho_{0}$ is constant density of particles, and $\Delta \mathbf{u}_{* *}$ is correction in velocity in modification step and considering the momentum equation is defined as below:

$\nabla U_{* *}=\frac{1}{\rho_{*}} \nabla P_{t+1} \Delta t$

Based on Eqs. 13 and 14, the pressure equation is as below:

$$
\nabla\left(\frac{1}{\rho^{*}} \nabla P_{t+1}\right)=\frac{\rho_{0}-\rho_{*}}{\rho_{0} \Delta t^{2}}
$$

\subsection{Solid Boundary and Free Surface}

In general, the solid boundary can be simulated by particles whose location, according to problem type, is fixed or variable in time. The particles prevent the penetration of the fluid particles close to solid 
boundaryin it by applying repulsion force on them.In other words, the fluid particles can be prevented from gathering near solid boundary by solving the Laplacian pressure equation (14) for solid particles. In addition, there are some particles out of solid boundary called virtual particles, the pressure of which can be calculated via applying Newman boundary conditions

\section{Results}

This problem is examined by smooth particles hydrodynamic method for the parameters, as shown in Table 1. The volute diameter is $5 \mathrm{~cm}$, the blade number is 8 and blade curvature is $30^{\circ}$. The $\mathrm{C}++$ code and tree algorithm are utilized to find adjacent points [8]. Firstly, the particles are arranged regularly and the velocity of $2000 \mathrm{rpm}$ is applied to particles of blade, as shown in Fig. 1. The total number of particles is 20000 and resolving time is 2 hours. Also, blood is considered as Newtonian fluid. All flow simulations and result analysis are visualized by Paraview software and results are shown for various time intervals. The blood tends to make vortexflow between two blades due to velocity gradient. The velocity is the leastat center and inlet of pump and between the blades and initial diameter it reaches to maximum. The velocity close to boundary is reduced and pressure is increased, consequently. In the inlet, velocity is less than $0.5 \mathrm{~m} / \mathrm{s}$, therefore this area has a potential for clotting. Therefore, some correction should be done on pump to prevent clotting. The velocity maximum mean is less than $4 \mathrm{~m} / \mathrm{s}$. Results of velocity distribution shown in fig. 2 demonstrate that blade (a) has a higher velocity distribution about $4 \mathrm{~m} / \mathrm{s}$. In input area,the blood velocity is less than $1.5 \mathrm{~m} / \mathrm{s}$ and is potentially dangerous for clotting. Also, vortex and disturbancein blade (a) is higher than other types of blade that in turn reduces the hydraulic efficiency of the pump.

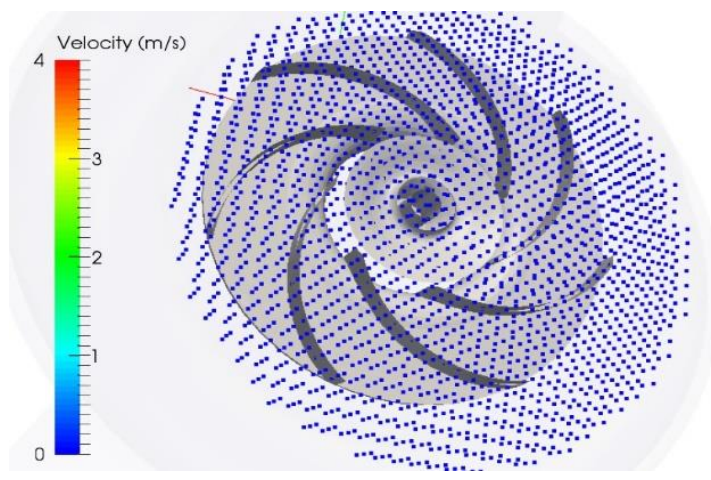

Figure 1. Particles Arrangement at $t=0$,Initial Pressure and Velocities are Considered Zero 


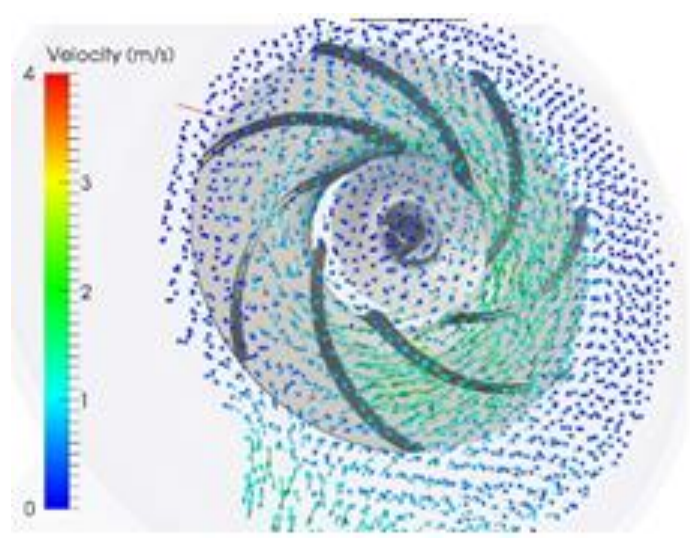

Figure 2.Particles Arrangement at $\mathrm{t}=0.2 \mathrm{~s}$, withlncreasing Velocities, Field Lines are Extended

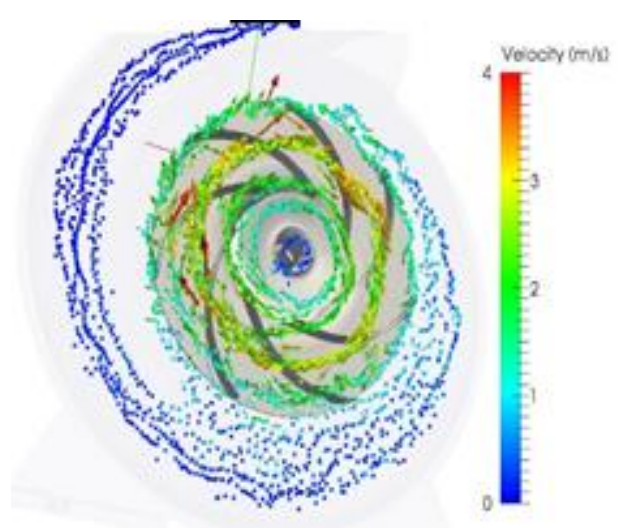

Figure 3.Flow Field at $t=2.2 \mathrm{~s}$, Total Velocity Average of Particles is $3.7 \mathrm{~m} /$

Using the diffuser can help flow circulation and preventliquid stagnation. The tongue area of pump, due to blocking the flow, makes disturbance and vortex and increases the time that blood is under shear stress. The space between blade and shell is also an appropriate place for return flow and shear stress on blood. It is necessary to determine the sufficient distance to increase the efficiency of the pump. The shear stress between two blades is shown in Fig. 4. 


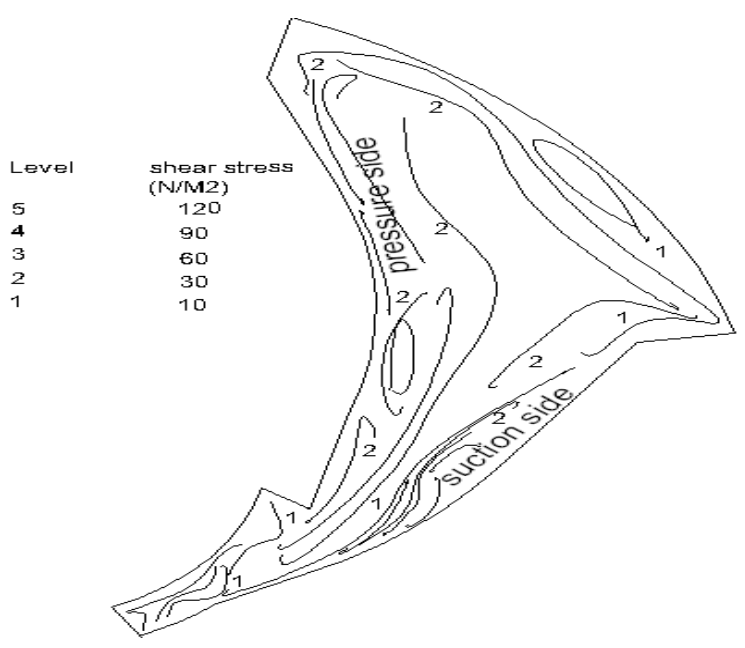

\section{Figure 4. Shear Stress Distribution between Two Blades Area}

The ultimate shear stress for hemolysis is $120 \mathrm{~N} / \mathrm{m} 2$ [8] and maximum shear stress in blades is $100 \mathrm{~N} / \mathrm{m} 2$. Although this value is less than hemolysis shear stress, it can deform the red blood cells and reduce their performance efficiency.

Table 1.MainCharacters of Simulation

\begin{tabular}{|c|c|}
\hline PARAMETERS & VALUES \\
\hline Time Step & $0.001 \mathrm{~s}$ \\
\hline Initial particles distance & $0.04 \mathrm{~mm}$ \\
\hline Smoothed Length & $\mathrm{h}=1.5 \mathrm{~L}_{0}$ \\
\hline Number of particles & 20000 \\
\hline
\end{tabular}

\subsection{Results Validation}

To validate the results of velocity distribution obtained from simulation, the results of Posa's work [7] and chao[4] is illustrated in Fig. $5 \& 6$ and is compared to current research ones.

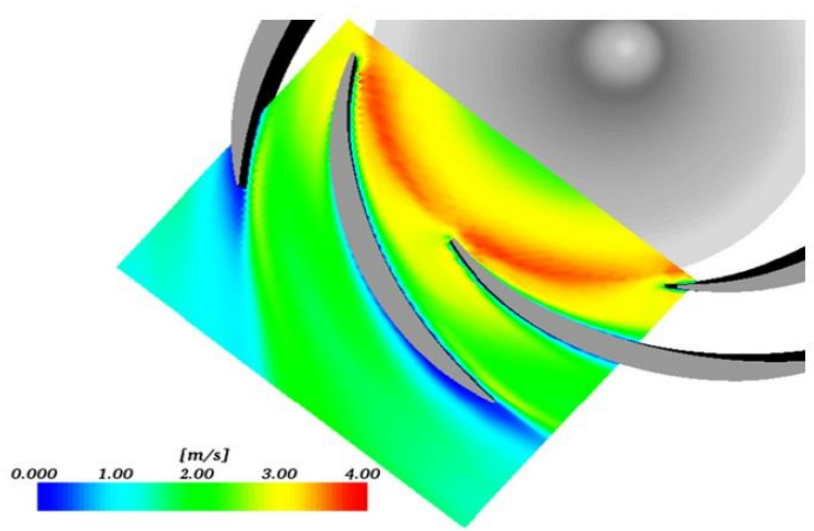

Figure 5: Velocity Distribution Base on Numerical Simulation by Posa [7] 


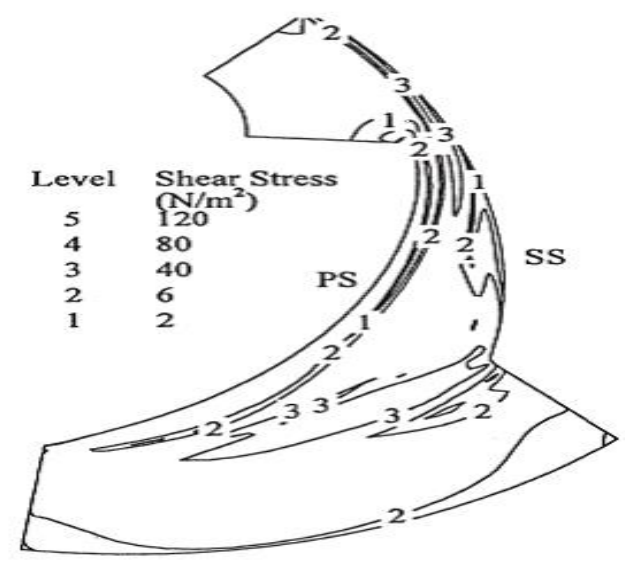

Figure 6.Shear Stress Distribution based on Finite Element Method by L.P. CHAU [4]

As shown in fig.5 and 6the result of simulation has good compatibility with other works in this field.

\section{Discussion}

The replacement or augmentation of failing human organs with artificial devices and systems has been an important element in health care for several decades. The pump performance is determined by examining its inner flow characteristics, which is undoubtedly the best method to improve the performance of pumps. There are a lot of geometrical and flow considerations in the artificial organs that are developed to perform in contact with the blood particles. For example high pressure and shear regions are important considerations in artificial organ design for preventing the stagnation. Recently, with the rapid progress in CFD and computer technology, the internal flow simulation has gradually become the important foundation of optimization and design for turbo-machinery. Maximummagnitude of velocities is shown in fig. 7. Since the axial velocities are negligible, it can be understood that 2D simulation is acceptable. Stagnation is also occurred in the inlet of pump which can be eliminated by geometrical modification. Maximum shear rate is seen near the blade at pressure side. The gap between impeller and volute is important which is considered about $1 \mathrm{~mm}$. Further researches can be done by considering different distance. 


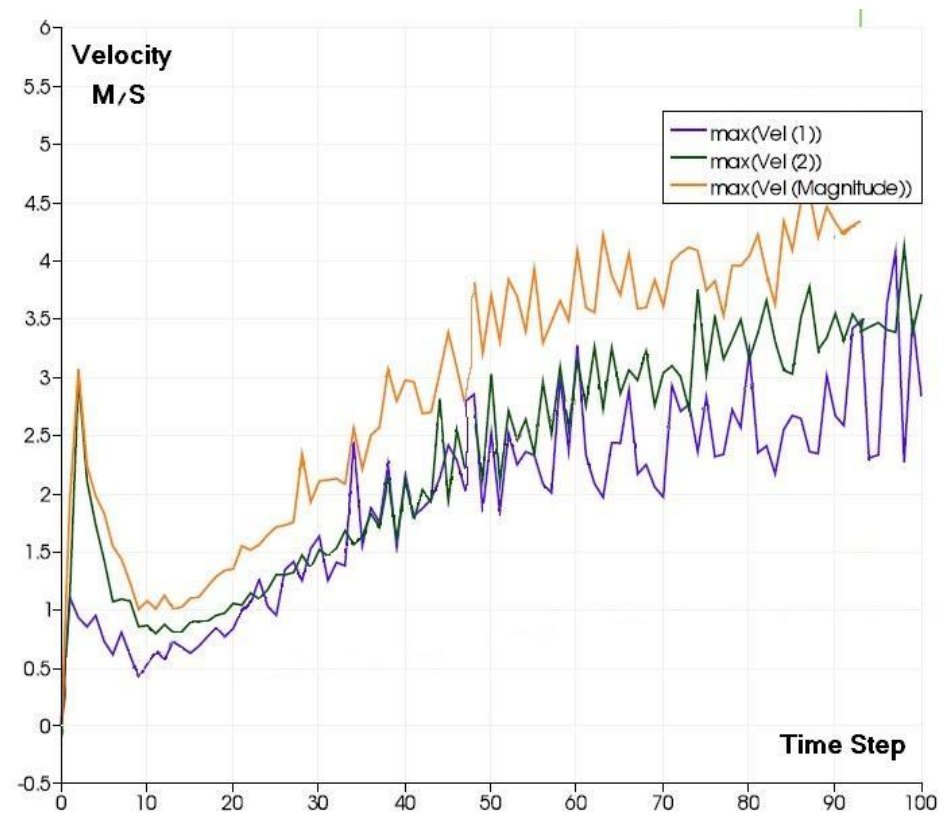

\section{Figure 7. Maximum Tangential Velocities (vel. 2) and Radial Velocities(vel.1) and Maximum Velocities are Shown per Each Time Step}

\section{Conclusion}

The smooth hydrodynamic particles method is utilized to simulate centrifugal heart pump. The blood flow patterns can show the blood damages by determining theareas of circulation flow and high pressure gradient and shear stress, although the amount of damage is not realized. Therefore, the possible damages can be reduced by evaluating the locations in which return flow or vertex occurs or disturbance is higher than other areas as well as points that may cause inertia and clotting due to low velocity. The location where rotational flow exists has attracted most of pump designers' concern to itself and it is because of blood blockage in pump, increased applied shear stress time and therefore consequent damages.In present paper, there is an ignorable difference between input fluid angle and input blade angle and no vortex is observed in the begining side of the blade. Also, due to pressure gradient in pressure side and suction side, the vortex is inevitable inthe end side of the blade. The flow lines in the blade are longer than shell flow lines that shows the higher velocity in the blade. There are few studies in this regards but the result from SPH method have good compatibility with other numerical solution methods[]. Areas susceptible to stagnation and clotting aredetermined in the inlet of the pump that shows a lower velocity in comparison with other pumps [5].Also utilizing this pump is proposed to reduce hemolysis probability in patients whose blood viscosity is considerably high and bear dangerous shear stress. 


\section{Acknowledgement}

We appreciate Dr. Amanipour for his precious comments in preparing this paper.

\section{References}

[1] N.Amanipour, "Principal of simulation based on SPH", Rasht,Publication of Gilan University,(2013).

[2] M. D.Sinnott and W. Paul, "CLEARY "Effect of rotor blade angle and clearance on blood flow through a non- pulstile, axial, neart pump", seventh international conference,(2009);Australia.

[3] L.P.Chaua and T.Akamatsu, "Measurment of gap pressure and wall shear stress of a blood pump model", Division of health Services research, NanyangTechnological University, Medical EnglneeringPhysics, (2011).

[4] S.C.M. Yu, B. T.H.Ng, W.K. Chan and L.P.Chau, "The flow patterns within the impeller passages of a centoifugal blood pump model", School of Mechanical and Production Engineering, Singapore Medical Engineering Physics, (2011) .

[5] F. H. Min, "Design of implantable axial- flow blood pumpand numerical studics on its performance", Slence Direct, Journal of hydrodynamics, Tongji University, (2012).

[6] M. V. Kadletz and E.Wolne, "Bio artificial surfaces- Blood surface interaction",Material Science and Engineering, Medical University of Vienna, (2011).

[7] A. Posa, A. Lippolis, R. Verzicco and E. Balaras, "Large-eddy simulations in mixed-flow pumps using an immersed-boundary method", Dipartimento di IngegneriaMeccanicae Gestionale, Politecnico di Bari, (2011).

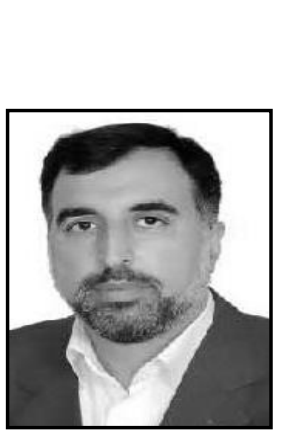

\section{Authors}

Mehdi Navidbakhsh, He is working as Associate Professor and Head in the Department of Biomechanic, Iran University of Science and Technology, which is a prestigious institution of Iran. He received his Ph.d. in energy conservation from Nancy University, French. He has 25 years of teaching experience with good academic record. He presented his papers in many seminars in National and International conferences. 60 papers of him were published in International Journals.

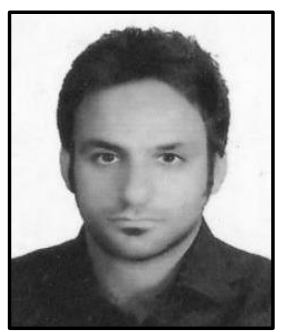

ErfanRahmanian, he received his M.s in biomechanic in the Department of biomechanic, iranyniversity of science and technology, tehran which is one of prestigious University in Iran. he has nearly 9 years of experience in various engineering fields. he has attended many seminars and presented his papers on various topics in National and International conferences. More than 10 papers were published in various national and International Journals. 